\title{
Comparison of thermal diffusivity measurement techniques
}

\author{
by F. Cernuschi ${ }^{1}$, P.G. Bison ${ }^{2}$, S. Marinetti ${ }^{2}$, \\ A. Figari ${ }^{1}$, L. Lorenzoni ${ }^{1}$, E. Grinzato ${ }^{2}$ \\ (1) CESI, via Reggio Emilia 39, 20090 Segrate (MI), Italy \\ (2) CNR-ITC sez. Padova, C.so Stati Uniti 4, 35127 Padova, Italy
}

\begin{abstract}
Thanks to the collaboration between two laboratories, we present different techniques to measure thermal diffusivity. A brief description of any technique both in the experimental layout and in the processing algorithms is given. Results obtained on samples cut from the same block of stainless steel AISI 304, are reported. Uncertainty evaluation of any measurement is reported as well.
\end{abstract}

\section{Introduction}

The knowledge of the thermal conductivity and/or the thermal diffusivity is more and more required in many industrial fields. As a matter of fact, these are the most important parameters when heat transfer processes are involved.

In the power generation industries, materials are selected primarily considering their thermal properties [1-4]. In the automotive and aeronautical industries, CMC (ceramic matrix composite) materials for high performance brakes and heat shields are under development and manufacturers require, as essential information the thermal diffusivity [5].

Notwithstanding the parameter involved in stationary heat conduction processes is the thermal conductivity $k$, it is a consolidated practice to experimentally evaluate the thermal diffusivity $\alpha$ by transient methods and to calculate indirectly the conductivity by the equation $k=\alpha \rho C$, where the specific heat $C$ and the density $\rho$ should be known. This approach has been often suggested because the thermal diffusivity measurement is usually less time consuming and more productive if compared with the stationary methods used for thermal conductivity measurements. The methods for the thermal diffusivity measurement proposed over the last four decades, belong mainly to photothermal and photoacoustic $[6,7]$ techniques, generally confined to the laboratories. In the last years, due to the significant progress in infrared technology as well as in electronics, some thermographic techniques for the in-situ thermal diffusivity evaluation have been developed [8-16].

In order to verify the advantages, the limitations as well as the uncertainty sources, the reliability and the accuracy of some of the photothermal and thermographic techniques, a Round Robin between the Istituto per le Tecnologie della Costruzione of the Italian National Research Council and CESI (the Research Centre of ENEL, the main Italian Electricity Company) has been launched. In particular, the experimental activity has been limited to some samples extracted from the same plate of the stainless steel AISI304. The final aim of this work is to show that some of these techniques could be considered as a standard for the thermal diffusivity measurements.

\section{The techniques}

\subsection{The laser flash}

The Laser Flash method is based on the analytical solution of the heat conduction problem within a infinite plate of thickness $L$, initially at a uniform temperature and heated 
on one of the two surfaces by a Dirac (i.e. instantaneous) energy pulse. The dimensionless temperature on the rear face of the plate as a function of time is:

$$
V(t)=\frac{T(L, t)}{T_{\infty}}=1+2 \sum_{n=1}^{\infty}(-1)^{n} \exp \left(-n^{2} \pi^{2} t / t_{c}\right)
$$

where $T_{\infty}=\frac{Q}{\rho C L}$ the equilibrium temperature, $Q$ the heating pulse intensity and $t_{c}=\frac{L^{2}}{\alpha}$. For evaluating the thermal diffusivity, the solution proposed originally by Parker et al. [17] consisted in using the following relation:

$$
\alpha=0.1388 \frac{L^{2}}{t_{1 / 2}}
$$

where $t_{1 / 2}$ is the time corresponding to the half maximum increase of $V(t)$. Relationships analogous to eq.(2) can similarly be obtained for times corresponding to different percentages of the maximum temperature increase [18]. An other alternative method consists in fitting the whole experimental data by eq.(1). The comparison between all these different method of data reduction carried out on a wide set of experimental results has shown a very good agreement when the experimental conditions fit well the theoretical assumptions [19].

The experimental system shown in fig. 1 uses a pulsed $1.06 \mathrm{~mm}$ wavelength $\mathrm{Nd}$ :YAG laser (Laser Metrics Winterpark FL-USA) for heating the sample. The pulse energy can be tuned in the range 8-50J. The beam shape is circular with a uniform intensity. Sample is located within a tantalum furnace with molybdenum shield (Theta Instruments. Port Washington, NY-USA) where it is possible to reach $1500^{\circ} \mathrm{C}$. The sample and the furnace are both inside a vacuum chamber and an infrared detector senses the temperature of the rear face of the sample through an infrared window. The signal is then amplified, acquired and processed by a PC. The experimental activity has been carried out on a $10 \mathrm{~mm}$ diameter disk shaped sample $1474 \mu \mathrm{m}$ thick. Measurements have been carried out five times for statistical reasons and the associated uncertainty is indicated together with the average result in table 1.

\subsection{The TWI method}

Thermal wave Interferometry is a well-established technique for the measure of the thermal diffusivity of coatings and thin slabs. As a matter of fact, the propagation of thermal waves with angular frequency $\omega$ generated within a two layers solid is affected by the interface between the first and the second layers. In particular thermal waves are partially reflected and transmitted at the separation surface of the two different materials like conventional waves. The interference between propagating and reflected waves alters the phase and the amplitude of the ac component of the surface temperature.

Table 1: Experimental and literature values of thermal diffusivity values for AISI304 stainless steel

\begin{tabular}{|l|c|c|}
\hline & Diffusivity $\left[\mathrm{cm}^{2} / \mathrm{s}\right]$ & Error [cm $\left.{ }^{2} / \mathrm{s}\right]$ \\
\hline Literature value (www.matls.com) & 0.040 & \\
\hline Laser Flash & $0.0399^{*} 10^{-4}$ & $\pm 0.0006^{*} 10^{-4}$ \\
\hline TWI & $0.040^{*} 10^{-4}$ & $\pm 0.001^{*} 10^{-4}$ \\
\hline $\begin{array}{l}\text { Thermographic method I (spatially resolved diffusivity } \\
\text { measurement) }\end{array}$ & $0.040^{*} 10^{-4}$ & $\pm 0.006^{*} 10^{-4}$ \\
\hline Thermographic method II (lateral thermal waves) & $0.0398^{*} 10^{-4}$ & $\pm 0.0006^{*} 10^{-4}$ \\
\hline Thermographic method III (single side flash method) & $0.0401^{*} 10^{-4}$ & $\pm 0.004^{*} 10^{-4}$ \\
\hline
\end{tabular}




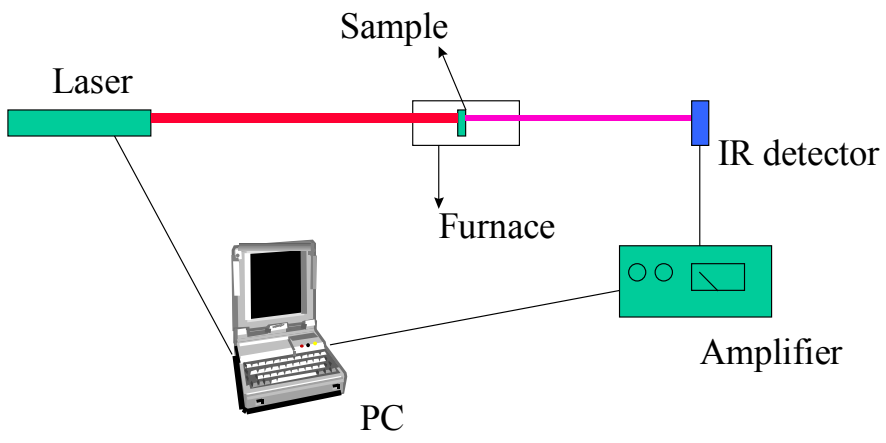

Fig. 1. Sketch of the experimental set up of the Laser Flash system

$$
A=\left(\frac{e^{4 h}+2 B e^{2 h} \cos (2 h)+B^{2}}{e^{4 h}-2 B e^{2 h} \cos (2 h)+B^{2}}\right)^{1 / 2} \quad \Delta \phi=\arctan \left(\frac{e^{-2 h}\left(e^{4 h}-B^{2}\right)}{2 B \sin (2 h)}\right)
$$

where $h=L / \mu$ is the normalised thickness of the first layer. $B=\frac{\varepsilon_{1}-\varepsilon_{2}}{\varepsilon_{1}+\varepsilon_{2}}$ (where $\varepsilon_{i}=\sqrt{\rho_{i} C_{i} k_{i}}$ is the thermal effusivity) is the thermal wave reflection coefficient at the interface where subscripts 1 and 2 refer to first and the second layer, respectively. $\mu=\sqrt{\frac{2 \alpha_{1}}{\omega}}$ is the thermal diffusion length and represents the depth where the initial magnitude of the thermal wave reduces by a $1 / e$ factor.

Experimentally, the evaluation of the thermal diffusivity $\alpha$ of the first layer can be performed by fitting the experimental data acquired at different modulation frequencies with one of the two expressions of eq.(3). Phase measurement is usually preferred to the amplitude one because it is less sensitive to the optical features of the sample surface as well as to laser power variation during the measurement. As an example, Fig. 2 shows theoretical curves for phase as a function of the normalised coating thickness $h$ for $R$ values ranging between -1 and 1 .

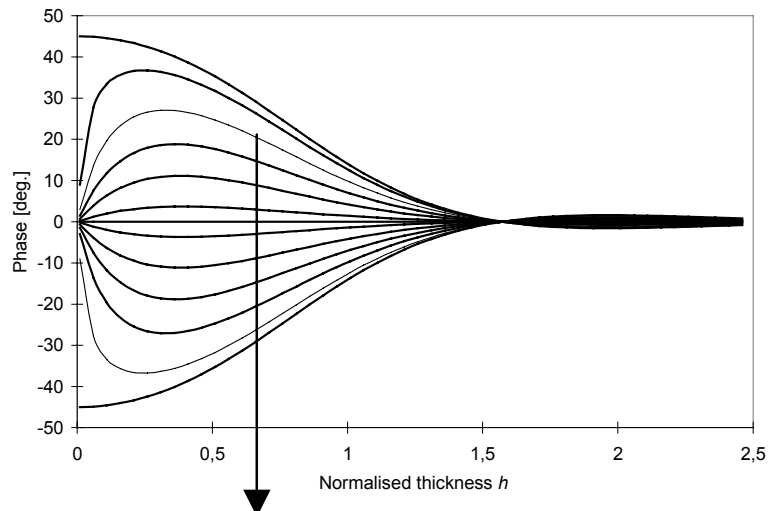

Fig. 2. Phase of the ac component of temperature versus the normalised thickness $h$ for different values of the reflection coefficient $R$ ranging from -1 to 1 (in the sense of the arrow) 


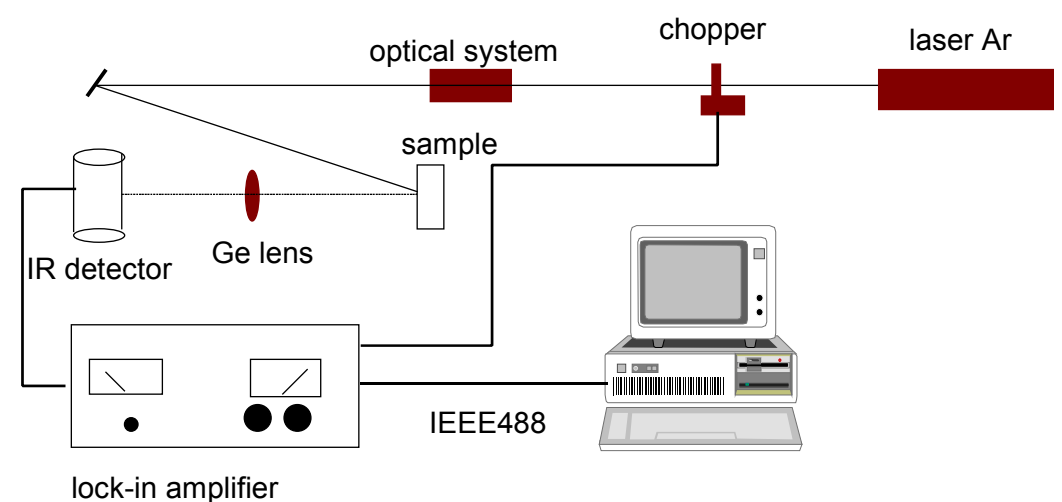

Fig. 3. TWI experimental set-up

Fig. 3 shows the TWI experimental set-up: the heating source is a $5 \mathrm{~W}$ Ar ion laser (Spectra Physics 2020). The intensity was modulated using a high stability mechanical chopper (HMS ElektroniK mod. 220-RG). The laser beam was expanded on the sample surface in order to guarantee the one-dimensional approximation. The monitoring of the ac-component of the surface temperature was performed by an $\mathrm{Hg} 1-\mathrm{xCdxTe}$ infrared detector (EG\&G Judson,). The signal was amplified by a dc-coupled low noise transimpedance preamplifier and than by a lock-in amplifier (EG\&G mod. 5501). A time averaging procedure with statistic treatment of the data and subsequent coating thermal diffusivity evaluation was performed under the control of a proprietary PC program.

In this specific case, measurements were carried out on a thin AISI304 slab $660 \mu \mathrm{m}$ thick modulating the heating source in frequency range $1-20 \mathrm{~Hz}$ as also shown in Fig. 4. The uncertainty associated to the thermal diffusivity value reported in table 1 is related to the standard deviation of three repeated measurements. More details of the experimental set up and measurement procedure are reported elsewhere [19].

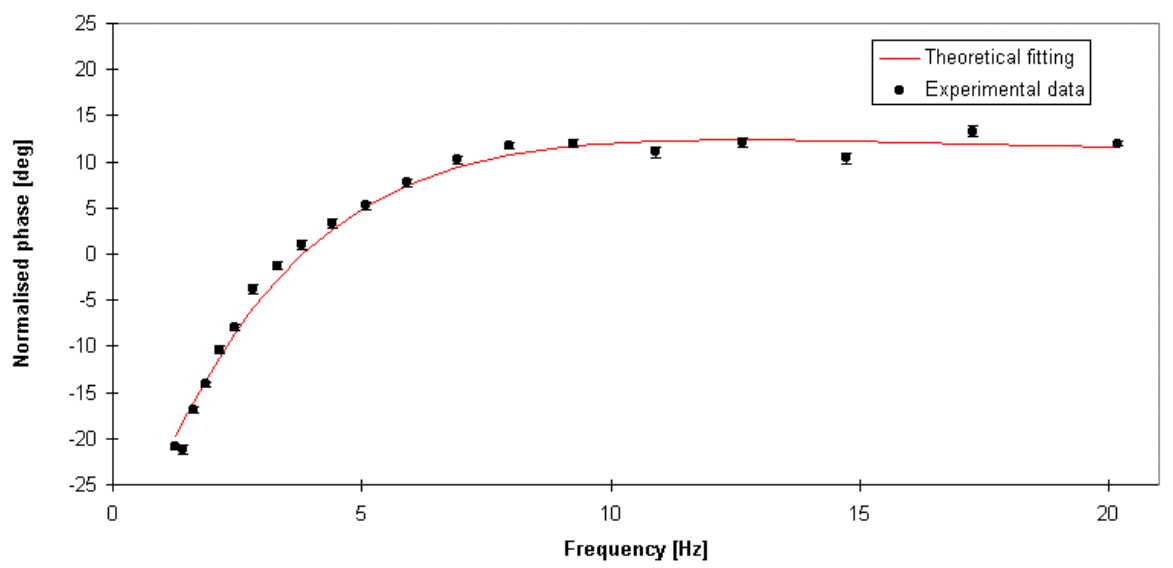

Fig. 4. Normalised phase of the ac component of the surface temperature versus the modulation frequency for the AISI304 sample 

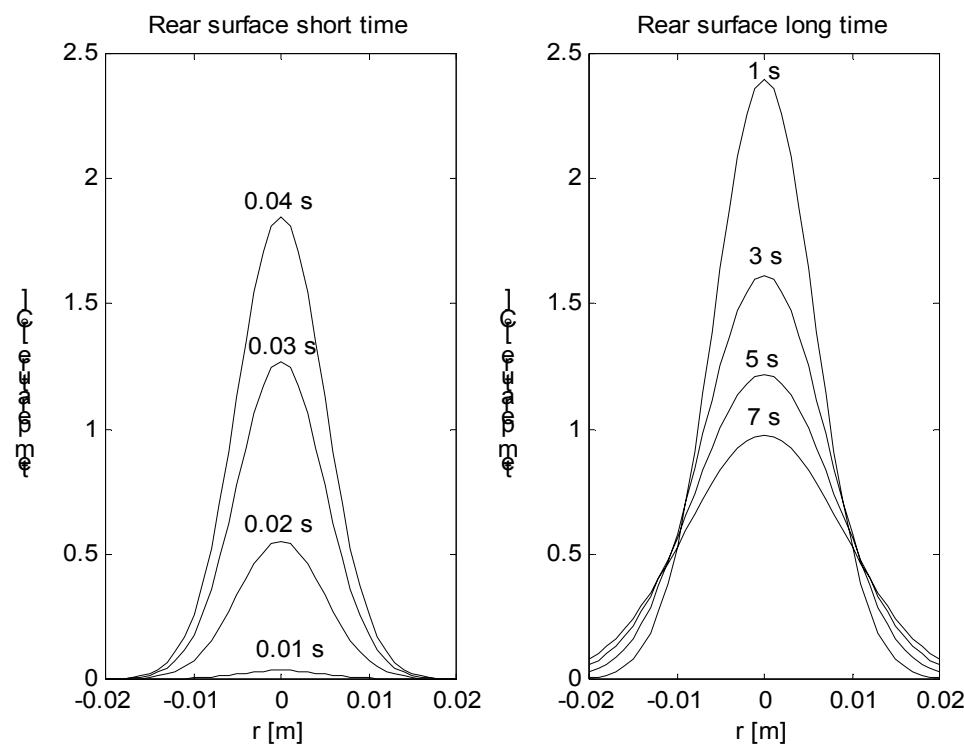

Fig. 5. Eq. (6). $T(r, L, t)$ as a function of the distance r. Curves refer to time values ranging from 0.01 to 7 seconds. Computations have been performed by using the energy $Q=1 \mathrm{~J}$ for a $5.0 \mathrm{~mm}$ diameter Gaussian spot.

\subsection{The Thermographic method I (spatially resolved method)}

The temperature on the rear $(z=L)$ surfaces of an infinite slab after an instantaneous spatially Gaussian shaped heating on the front $(z=0)$ is [15]:

$$
T(r, L, t)=\frac{2 Q}{\varepsilon \sqrt{\pi^{3} t}} \sum_{n=-\infty}^{\infty} \exp \left(-\frac{((2 n-1) L)^{2}}{4 \alpha t}\right) \frac{1}{\left(R^{2}+8 \alpha t\right)} \exp \left(-\frac{2 r^{2}}{R^{2}+8 \alpha t}\right)
$$

Fig. 5 shows the spatial distribution of the temperature as a function of the distance $r$ from the spot centre for the rear slab surface at different times. Computations have been performed by using the thermal effusivity and thermal diffusivity values of $\mathrm{AISI} 304$ stainless steel $\left(\varepsilon=8049 \mathrm{~J} / \mathrm{m}^{2} \mathrm{~s}^{1 / 2} \mathrm{~K} \alpha=0.04^{*} 10^{-4} \mathrm{~m}^{2} / \mathrm{s}\right)$ and fixing the beam radius $R=5 \mathrm{~mm}$ and slab thickness $L=1 \mathrm{~mm}$. The term:

$$
\frac{1}{2 \pi} \frac{1}{\left(R^{2}+8 \alpha t\right)} e^{-\frac{2 r^{2}}{R^{2}+8 \alpha t}}
$$

in eq.(4) is related to the finite size of the heating beam and it is used for the measurement of the in-plane thermal diffusivity. In particular the proposed technique consists in best fitting, at some times, the spatial profile of the temperature along a line passing through the centre of the heated spot on the rear sample surface (but a similar procedure could be followed for the front surface as well) by using a Gaussian function; in fact the shape of the temperature profile at every fixed time depends only on the Gaussian term in Eq.(5). The angular coefficient of the straight line describing the Gaussian widening as a function of time is eight times the thermal diffusivity of the material: 


$$
b^{2}=R^{2}+8 \alpha t
$$

Note that neither initial time nor beam radius nor sample thickness estimation were required.

The experimental set-up (see Fig. 6) consists of a 1000W continuous lamp (PSC 1000 ILC Technology Inc. CA) electronically shuttered as heating source and of an infrared Jade Focal Plane Array camera (CEDIP, France) sensitive in the spectral range $8-10 \mathrm{~mm}$ for monitoring the surface temperature distribution of the sample. The sample was a $30 \times 30 \mathrm{~cm}$ AISI304 stainless steel plate $1.7 \mathrm{~mm}$ thick. The result and its uncertainty from several tests are reported in table 1.

\subsection{The Thermographic method II (lateral thermal waves)}

The thermal model adopted to describe the proposed experimental set-up is the original one due to Ångström [20,21]. It describes the temperature along a semi-infinite bar heated by a periodic source on one end, exchanging heat with the environment and being thermally thin. Therefore temperature varies only down to the bar (1D diffusion) according to the following equation:

$$
\begin{aligned}
& \Delta T(x, t)=\sum_{n=0}^{N} A_{n} \exp \left(-k_{1 n} x\right) \sin \left(n \omega t-k_{2 n} x+\psi_{n}\right) \\
& \Delta T(x, t)=T(x, t)-T_{\text {env }} v=\frac{h p}{S c_{p} \rho} \\
& k_{1 n}=\sqrt{\frac{v+\sqrt{v^{2}+n^{2} \omega^{2}}}{2 \alpha}} k_{2 n}=\sqrt{\frac{v-\sqrt{v^{2}+n^{2} \omega^{2}}}{2 \alpha}}
\end{aligned}
$$

where $T(x, t)$ is temperature function depending on $x$ coordinate along the bar and time $t$, $T_{\text {env }}$ the environment temperature, $A_{n}$ the amplitude of the $n_{\text {th }}$ harmonic component, $\omega$ the angular frequency, $\alpha$ is the thermal diffusivity, $h$ heat exchange coefficient, $p$ and $S$ perimeter and cross-section area of the bar respectively, $c_{p}$ and $\rho$ specific heat and density, and $\psi_{n}$ the initial phase of the $n_{t h}$ harmonic component.

The goal of the data reduction procedure consists in determining the spatial phase velocity $k_{2 n}$ and the attenuation coefficient of the harmonic component $k_{1 n}$. Hence the diffusivity $\alpha$ and heat exchange related parameter $v$ are given by:

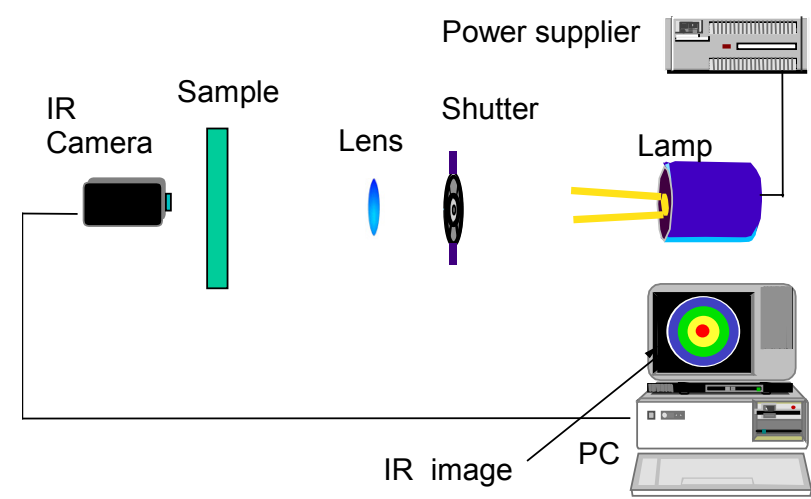

Fig. 6. Experimental set-up of the thermographic method for inplane thermal diffusivity measurement 


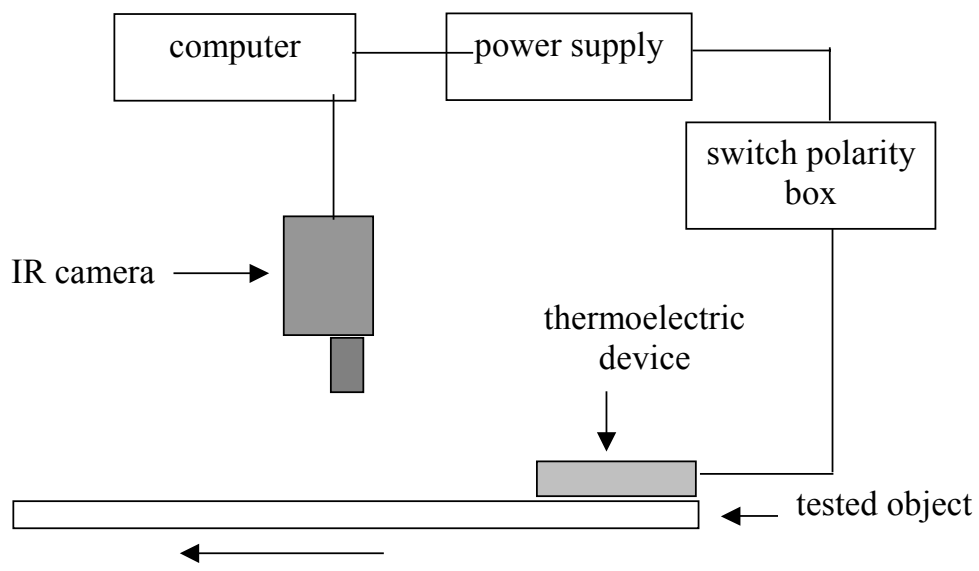

1D propagation of thermal wave

Fig. 7. Experimental set up for diffusivity measurements by thermoelectric generated thermal waves

$$
\alpha=\frac{n \omega}{2} \frac{1}{k_{1 n} k_{2 n}} v=\frac{n \omega}{2} \frac{k_{1 n}^{2}-k_{2 n}^{2}}{k_{1 n} k_{2 n}}
$$

A slab of AISI 304 with dimension $40^{*} 250^{*} 1.5 \mathrm{~mm}$ is tested. Its height $(40 \mathrm{~mm})$ is equal to the side of a thermoelectric device $(40 * 40 \mathrm{~mm})$ that is in contact with the surface on one end of the bar. Driven by a power supply controlled by a PC, it generates the thermal waves. After some cycles the periodic steady regime is reached and the temperature of the surface is grabbed through the IR camera. Fig. 7 shows the experimental lay-out. The acquisition is repeated at a regular time interval for some periods of the thermal wave. Temperature is finally sampled for each pixel along the propagation path of the thermal wave. As shown in Fig. 8 the sinusoidal temperature is dumped and phase shifted as the space position increases. The first step of the data reduction procedure consists in time fitting these sine temperature data obtaining amplitude and phase that depend on space. After that, a second fit along the space is done on both amplitude and phase giving finally the dumping factor and the phase velocity of the thermal wave. Measurements where done at different periods of the harmonic heating/cooling flux, and they are condensed in table 1. 


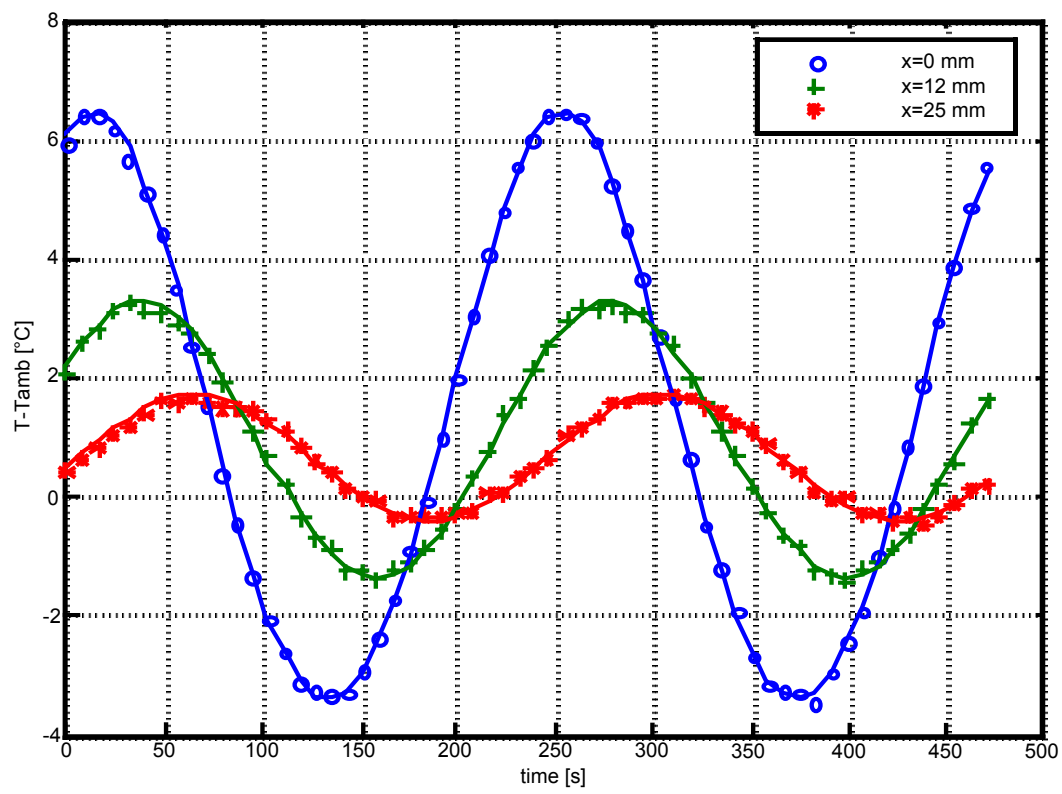

Fig. 8. Temperature vs time at increasing distances from the source/sink thermoelectric device, with fitting function superimposed (continuous line)

\subsection{The Thermographic method III (single side flash method)}

Diffusivity can be measured in reflection mode by flash heating. The solution for the heated side of the slab is that given in eq. (1) but for the term $(-1)^{n}$. For the rear side it gives the alternate signs of the series terms making the solution increasing monotonically. For the front side the lack of such a term makes the solution decreasing monotonically from the maximum temperature value after the flash to the $T_{\infty}$ value. Unfortunately the temperature reached during or immediately after the flash is not a reliable value to measure and therefore we cannot extract a noticeable time from the amplitude evolution much like in eq. (2). On the other end it is possible to show that multiplying the solution of the front side by the cube root of the time, such a function presents a minimum (that can be measured more easily) at $F o=0.2656\left(F o=\alpha t / L^{2}\right)$ giving the following relation for the diffusivity measurement [25]:

$$
\alpha=\frac{0.2656 L^{2}}{t_{\min }}
$$

The experimental set-up is shown in fig. 9. Two photographic flash lamps, $4800 \mathrm{~J}$ nominal energy delivered in about $5 \mathrm{~ms}$, heat one side of the AISI $304 \mathrm{slab}, 1.51 \mathrm{~mm}$ thick. Temperature is grabbed along a line on the heated surface from an AGEMA $900 \mathrm{LW}$ system for about $2 \mathrm{~s}$ with a sampling frequency of $2550 \mathrm{~Hz}$. Fig. 10 shows the plot of the function obtained multiplying the experimental temperature by the cube root of time. Results and related error obtained by error propagation of eq. (9) is reported in table 1.

\section{Conclusion}

Among the five methods here presented the Laser Flash has been assumed as reference because it is the only one covered by a standard [22-24] and using a 
commercial equipment. From the point of view of precision the thermographic method II (lateral thermal waves) gives the possibility to produce thermal waves of large wavelength thank to the Peltier driven heat flux generation. That permits to exploit the capabilities of modern thermographic camera in term of geometric resolution. TWI, the thermographic method III (one side flash) and the thermographic method I (space-resolved diffusivity measurement) are very interesting for their practical feasibility especially in situ, remaining at an acceptable level of precision.

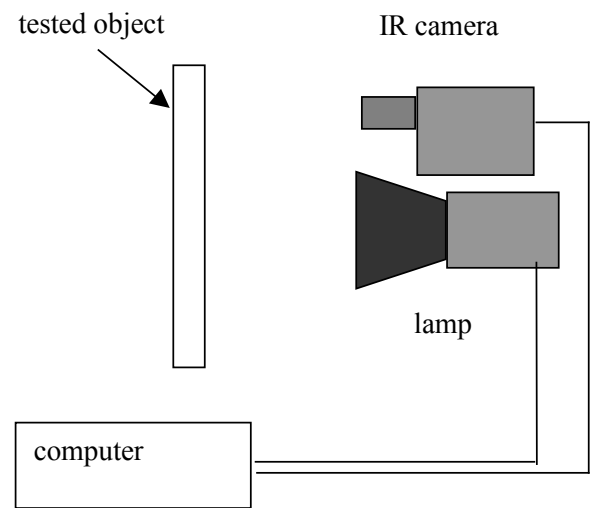

Fig. 9. Experimental set-up for one side diffusivity measurement by flash heating

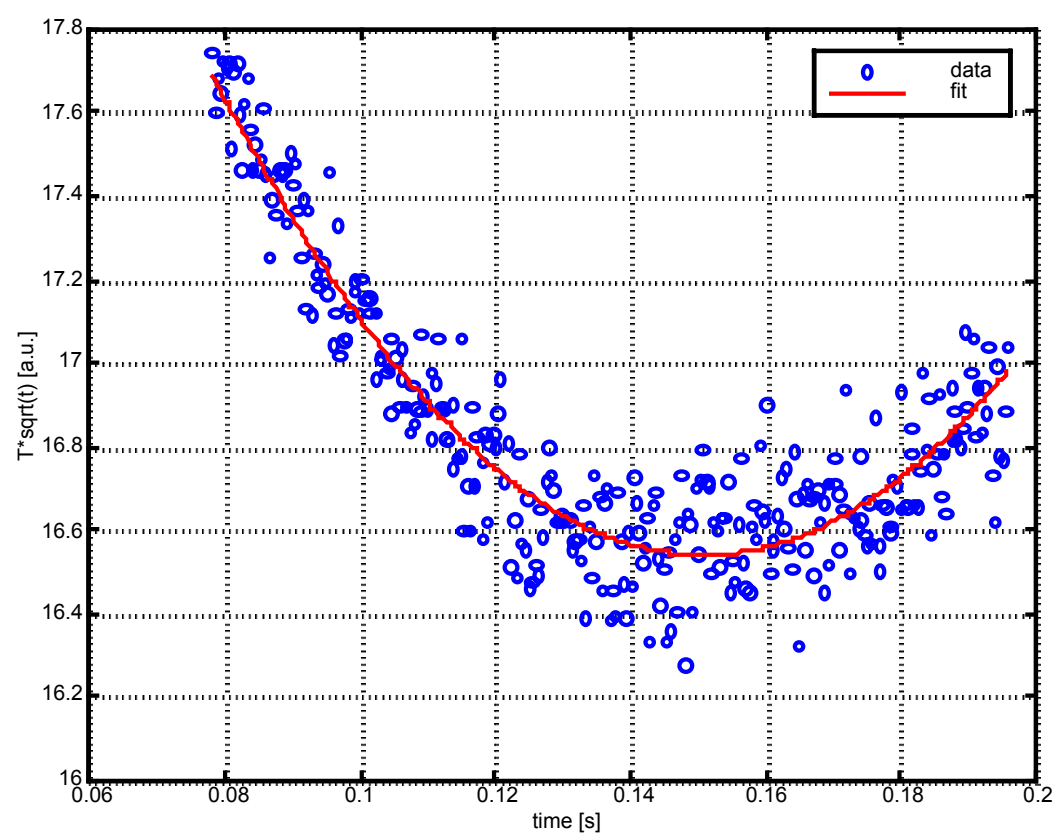

Fig. 10. Data and fitting function to obtain the time of minimum for diffusivity measurement 


\section{Acknowledgement}

This work has been partially developed within the frame of "Ricerca di Sistema" D.L. MICA 26/01/2000.

\section{REFERENCES}

[1] V.P. SWAMINATHAN and N.S. CHERUVU, "Gas Turbine Hot-Section Materials and Coatings in Electric Utility Applications", in Advanced Materials and Coatings for Combustion Turbines, Edited by V.P. Swaminathan and N.S. Cheruvu. ASM International, 1994.

[2] European Brite-Euram Project BRPR-CT97-0426 EFCC/UHTHE/B3

[3] European Brite-Euram Project BRPR-CT97-0426 EFCC/UHTHE/B4

[4] A. DONATO, A. ORTONA, C.A. NANNETTI, S. CASADIO, SiC/SiC Fibre Ceramic Composite for Fusion Application: a new Manufacturing Process Proc. 19th Symp. on Fusion technology (SOFT), Lisbon, (P) Sept. 1996.

[5] TWI Bulletin Nov/Dec. 1996 Reprint 508/6/96

[6] A. MANDELIS, Progress in Photothermal and Photoacoustic Science and Technology Vol. 1 (Elsevier 1991).

[7] D.P. ALMOND, P.M. PATEL, Photothermal Science and Techniques (Chapman \& Hall, London, 1996).

[8] J.C. KRAPEZ, in Proceedings of the 5th Workshop on Advanced Infrared Technology and Applications, 29-30 September 1999 Venice, Italy.

[9] I. PHILIPPI, J.C. BATSALE, D. MAILLET, and A. DEGIOVANNI, Rev. Sci. Instrum. 66 (1) 182 (1995).

[10] ZHOUNG OUYANG, L.D. FAVRO, and R.L. THOMAS in AIP Conference Proceedings $n^{\circ} 463$ Photoacoustic and Photothermal Phenomena, Rome, August 1998, edited by F. Scudieri and M. Bertolotti (AIP Woodbury, New York,1999) p. 374.

[11] C. S. WELCH, D.M. HEALTH, and W. P. WINFREE, J. Appl. Phys. 61 (3) 895 (1987)

[12] F. CERNUSCHI, L. FABBRI and M. LAMPERTI. in AIP Conference Proceedings $\mathrm{n}^{\circ}$ 463 Photoacoustic and Photothermal Phenomena, Rome, August 1998, edited by F. Scudieri and M. Bertolotti (AIP Woodbury, New York,1999) p. 392.

[13] D. HE, Y. GU, M. ZHENG and D. ZHU, 9th Int. Topical Meeting on Photoacoustic and Photothermal Phenomena, Nanjing (China), 27-30 June 1996, S.Y. Zhang Ed., Progress in Natural Science, pp. 169., 1996.

[14] T. YAMANE, S. KATAYAMA, M. TODOKI, Rev. Sci. Instrum. 67, (12) 1996, pp.4261.

[15] F. CERNUSCHI, A. RUSSO, L. LORENZONI, A. FIGARI, Rev. Sci. Instrum. 72, (19) 2001, 3988-3995.

[16] P.G. BISON, S. MARINETTI, A. MAZZOLDI, E. GRINZATO, C. BRESSAN, Crosscomparison of thermal diffusivity measurements by thermal methods, Infrared Physics and Technology, Vol. 43 N. 3-5, 2002.

[17] W.P. PARKER, R.J. JENKINS, C. P. BUTTLER and G.L. ABBOTT, J. Appl. Phys., 32, pp.1679, (1961).

[18] R.E. TAYLOR, K.D. MAGLIC, Compendium of Thermophysical Property Measurement Methods Vol: 1: Survey of Measurement Techniques, Eds. K.D. Maglic et Al. (Plenum Press New York 1984).

[19] F. CERNUSCHI, A. FIGARI, L. FABBRI, Journal of Materials Science 35, (2000), 5891-5897.

[20] M.A. ÅNGSTRÖM, Philos. Mag. 25, 130 (1863); Ann. Phys. (Leipzig) 114, 513 (1861) 
[21] H.S. CARSLAW and J.C. JAEGER, Conduction of heat in solids, Oxford University Press, London, 1959

[22] ASTM C714-72 Standard test method for thermal diffusivity of carbon and graphite by a thermal pulse method, (1972) ASTM.

[23] BS7134: Section 4.2: 1990; Method for the determination of thermal diffusivity by the laser flash (or heat pulse) method. British Standards Institution, (1990).

[24] JIS R 1611: Testing methods of thermal diffusivity, specific heat capacity and thermal conductivity for high performance ceramics by laser flash method, Japanese Standards Association (1991).

[25] V. VAVILOV, private communication. 